\title{
Three-isotope plot of fractionation in photolysis: A perturbation theoretical expression
}

\author{
M. K. Prakash ${ }^{\text {a) }}$ and R. A. Marcus ${ }^{\text {b) }}$ \\ Noyes Laboratory of Chemical Physics MC 127-72, California Institute of Technology, Pasadena, \\ California 91125
}

(Received 20 June 2005; accepted 9 September 2005; published online 31 October 2005)

\begin{abstract}
The slope of the three-isotope plot for the isotopomer fractionation by direct or nearly direct photodissociation is obtained using a perturbation theoretical analysis. This result, correct to first order in the mass difference, is the same as that for equilibrium chemical exchange reactions, a similarity unexpected a priori. A comparison is made with computational results for $\mathrm{N}_{2} \mathrm{O}$ photodissociation. This theoretical slope for mass-dependent photolytic fractionation can be used to analyze the data for isotopic anomalies in spin-allowed photodissociation reactions. Earlier work on chemical equilibria is extended by avoiding a high-temperature approximation. (C) 2005 American Institute of Physics. [DOI: 10.1063/1.2102908]
\end{abstract}

\section{INTRODUCTION}

Isotopomer fractionation for chemical equilibrium systems is described in the classic papers by Bigeleisen and Mayer ${ }^{1}$ and Urey. ${ }^{2}$ When the isotopic effect for the chemical systems is in equilibrium or otherwise follows the conventionally described effect, it is termed as mass dependent. The slope of three-isotope plots (defined below) for the massdependent oxygen isotopic fractionation in various thermal processes is about $0.52 .{ }^{3}$ Slopes that are substantially different from the mass-dependent value are usually referred to in the literature as anomalous mass dependent or mass independent. In some rare and interesting cases a striking mass independence with a slope of unity ${ }^{4,5}$ has been found.

Mass-dependent fractionation of isotopomers occurs in various geochemical processes. ${ }^{6}$ The deviations from such mass-dependent behavior are one source of information on geochemical processes. ${ }^{5,7}$ The three ways of presenting the isotopic fractionation information are as follows: (1) give the fractionation ${ }^{Q} \delta$, the relative rate of reaction of one isotopomer $Q$ relative to another or (2) construct a three-isotope plot by plotting the $\delta$ value of one isotopomer against another, each $\delta$ value being relative to the same reference isotopomer or (3) give the enrichment ${ }^{Q} \Delta$, which represents the difference of the measured fractionation ${ }^{Q} \delta$ of an isotope $Q$ from the expected mass-dependent value calculated from the normal isotope. Unless the fractionation is large, the threeisotope plot shows a linear relation between the fractionation of the two isotopomers relative to the third. The slope of this line depends upon the isotopic masses, and can depend on the fractionation of the geochemical process which induces the observed enrichment. The ${ }^{Q} \delta$ 's and ${ }^{Q} \Delta$ 's are widely used in atmospheric chemistry.

Deviations of ${ }^{Q} \Delta$ from zero are considered as anomalies ${ }^{7}$ and to identify anomalies a mass-dependent value appropri-

\footnotetext{
${ }^{a)}$ Electronic mail: meher@caltech.edu

b) Author to whom correspondence should be addressed. Electronic mail: ram@caltech.edu
}

ate to the process should be used. We note that kinetic processes can have a mass-dependent value different from that for chemical equilibria. ${ }^{8}$ For example, in collisions with a surface the dependence on mass varies as $1 / \sqrt{m}$, where $m$ is the mass of the colliding particle, while in Bigeleisen-MayerUrey expression, which contains vibrational partition functions, it varies as $1 / \mathrm{m}$.

Isotopomer fractionation also occurs in photodissociation reactions, a process important to atmospheric chemistry. The observed enrichment is explained in terms of the enrichments and magnitudes of the various sources of a given gas and the fractionations and magnitudes of the various loss mechanisms. In this way the enrichments in atmospheric samples can be used to study and quantify specific generating processes.

Using the theory of nearly direct photodissociation described later, we computed the fractionation factor, as defined in Sec. II A, of oxygen isotopes in the photolysis of $\mathrm{N}_{2} \mathrm{O}$ at different wavelengths. ${ }^{9}$ In the present paper we present these wavelength-dependent fractionation factors as a three-isotope plot. To our surprise the slope of the plot was the same as the mass-dependent value predicted by Bigeleisen-Mayer theory for a purely chemical equilibrium system. This similarity is quite unexpected a priori since the two processes are different and the functional dependence of the fractionation factors on the mass can be different for kinetic and chemical equilibrium processes. The present perturbation theoretical work on an expression for the slope of a three-isotope plot for photodissociation was undertaken to understand this computational result and for applications to atmospheric and laboratory systems.

\section{ANALYSIS}

\section{A. Photodissociation theory}

In the absorption of light in the UV or visible region, leading to electronic transitions in molecules, a transition frequently occurs to a repulsive electronic state of the mol- 
ecule and a direct photodissociation results. The detailed theory for the calculation of photodissociation cross sections is available. ${ }^{10,11}$ The molecule undergoes a direct dissociation when the lifetime of the molecule in the excited electronic state is less than or comparable to a vibrational period of the molecule, and an indirect dissociation when it is much longer. Long lifetimes in the excited state lead to the wellknown prominent structure in the absorption cross section ${ }^{10}$ and hence the presence of a more diffuse structure can be considered as a nearly direct-dissociation problem. ${ }^{12}$

The absorption cross sections for direct and nearly direct photodissociations can be obtained by a simple analysis using the multidimensional reflection principle. ${ }^{11,13}$ We use a form of the reflection principle ${ }^{10}$ for the absorption cross section $\sigma$ at a frequency $\omega$ which is more accurate than an earlier ${ }^{11,13}$ version of the principle. There are several expressions in the literature ${ }^{10,13}$ and the one we found ${ }^{9}$ to represent the absorption cross section $\sigma$ data best for a spin-allowed transition is given by ${ }^{10}$

$$
\begin{aligned}
\sigma_{i \nu}(\omega)= & \frac{\pi \omega}{3 \hbar \epsilon_{0} c} \int\left|\Psi_{\nu}(\mathbf{Q})\right|^{2}\left|\mu_{f i}(\mathbf{R})\right|^{2} \\
& \times \delta\left(\omega-\left(V_{f}(\mathbf{R})-\frac{1}{2} E_{i \nu}\right) / \hbar\right) d \mathbf{Q},
\end{aligned}
$$

where $\mathbf{R}$ denotes internal coordinates and $\mathbf{Q}$ the normal coordinates. $\mu_{f i}(\mathbf{R})$ is the transition dipole moment function for a transition between the ground and the excited electronic states $i$ and $f$, respectively, $V_{f}(\mathbf{R})$ is the potential-energy surface of the excited electronic state, and $E_{i v}$ is the energy of the initial nuclear vibrational state $\nu .\left|\Psi_{\nu}(\mathbf{Q})\right|^{2} d \mathbf{Q}$ is the probability density of the initial nuclear vibrational state $\nu$ in the ground electronic state $i$. For the latter we use as a zeroth-order approximation the harmonic-oscillator wave functions corresponding to the normal coordinates $\mathbf{Q}$.

The difference in the absorption cross sections of various isotopomers leads to a fractionation of the isotopomers during photodissociation. The rate of photodissociation for each isotopomer is given as $J(\omega)=\sigma_{\text {total }}(\omega) I(\omega) \phi(\omega)$, where $I(\omega)$ is the intensity of the incident light at frequency $\omega ; I(\omega)$ is independent of the molecule. $\phi(\omega)$ is the quantum yield of the reaction at that $\omega$ and is usually unity for direct or nearly direct photodissociation, and $\sigma_{\text {total }}$ is the total absorption cross section obtained by adding the contributions from all vibrational states in the ground electronic state, weighted by their thermal populations. The fractionation factor is dependent upon the ratio of the rates of photodissociation of two isotopomers, say $A$ and $A^{\prime}$ as

$$
\epsilon^{\left(A^{\prime}, A\right)}=\frac{J^{\left(A^{\prime}\right)}(\omega)}{J^{(A)}(\omega)}-1=\frac{\sigma^{\left(A^{\prime}\right)}(\omega)}{\sigma^{\left(A^{\prime}\right)}(\omega)}-1
$$

As expected from Eq. (1) the fractionation factor arises mainly from isotopic differences in the wings of the wave function, and so is least at the absorption maximum. Indeed, using $\mathrm{N}_{2} \mathrm{O}$ as an example, Yung and Miller ${ }^{14}$ found zero fractionation near the absorption maximum of $185 \mathrm{~nm}$.

\section{B. Slope of the three-isotope plot}

In the present note we derive a perturbation theoretical expression for the slope of the three-isotope plot of $\epsilon^{\left(A^{\prime \prime}, A\right)}(\omega)$ vs $\epsilon^{\left(A^{\prime}, A\right)}(\omega)$, for three isotopomers $A, A^{\prime}$, and $A^{\prime \prime}$. Our derivation of the perturbation theoretical expression is as follows: in evaluating the integral in Eq. (1), the Q's are transformed into mass-independent internal coordinates $\mathbf{R}$, because the fact that $V_{i}(\mathbf{R}), V_{f}(\mathbf{R})$, and $\mu_{f i}(\mathbf{R})$ are independent of isotopic substitutions simplifies the analysis. The mass effects occur in the vibrational frequencies, the normal coordinates $\mathbf{Q}$ in the ground electronic state vibrational wave functions, and in the energies $E_{i v}$. The method used to relate $\mathbf{Q}$ in the $|\Psi(\mathbf{Q})|^{2} d \mathbf{Q}$ to internal coordinates $\mathbf{R}$ depends functionally on the $G$ matrix $^{9,15}$ and the latter depends on masses in the functional form of reciprocal masses $1 / m_{i}$. Similarly the vibrational frequency and the energy $E_{i \nu}$ depend in a rather complicated way on $G$ and hence on $1 / m_{i}$ 's. In summary, the expression for the absorption cross section is a function of reciprocal masses $1 / m_{i}$, i.e., $\sigma_{\text {total }}$ $=\sigma_{\text {total }}\left(\omega, 1 / m_{1}, 1 / m_{2}, \ldots\right)$. To find the first-order isotopic perturbation in $\sigma_{\text {total }}$, a Taylor series expansion of $\sigma_{\text {total }}$ is used to the first order. The perturbation treatment for the entire expression of the cross section when the mass $m_{i}$ is isotopically substituted involves a differentiation of the expression for the cross section with respect to $1 / m_{i}$ and multiplied by the first-order difference $\Delta\left(1 / m_{i}\right)=1 / m_{i}^{\left(A^{\prime}\right)}$ $-1 / m_{i}^{(A)}$ in the two isotopes, i.e.,

$$
1+\epsilon^{\left(A^{\prime}, A\right)}(\omega)=\sigma^{\left(A^{\prime}\right)} / \sigma^{(A)} \approx\left(1+\sum_{i} \frac{\partial \sigma^{(A)}}{\partial\left(1 / m_{i}\right)} \Delta\left(1 / m_{i}\right)\right),
$$

where the subscript total is omitted and where the sum is over all the masses that are isotopically substituted. The factor $\partial \sigma^{(A)} / \partial\left(1 / m_{i}\right)$ that is common to both the abscissa and the ordinate has no effect on the slope of the three-isotope plot. Thus, the slope $\beta$ of the $\epsilon^{\left(A^{\prime \prime}, A\right)}(\omega)$ vs $\epsilon^{\left(A^{\prime}, A\right)}(\omega)$ plot based on the above perturbation method is obtained as

$$
\beta=\left(1 / m_{i}^{\left(A^{\prime \prime}\right)}-1 / m_{i}^{(A)}\right) /\left(1 / m_{i}^{\left(A^{\prime}\right)}-1 / m_{i}^{(A)}\right)
$$

and

$$
\epsilon^{\left(A^{\prime \prime}, A\right)}=\beta \epsilon^{\left(A^{\prime}, A\right)} .
$$

Thus, in this perturbation treatment of the three-isotope plot, it is the form $(1 / \mathrm{m}$ in the above case) in which the masses appear in the function, rather than the function itself that is important.

Like the isotopic fractionation in chemical equilibrium the present isotopic fractionation in the photolysis is a quantum effect: if the probability distribution in the coordinates $\left|\Psi_{\nu}(\mathbf{R})\right|^{2}$ was replaced by a thermally equilibrated classical Boltzmann probability distribution that is proportional to $\exp \left(-V_{i}(\mathbf{R}) / k_{B} T\right)$, the result for $\sigma_{\text {total }}$ in the reflection principle approximation would be 
$\sigma_{\text {total }}(\omega)=\frac{\pi \omega^{2}}{3 \epsilon_{0} c k_{B} T} \frac{\int e^{-V_{i}(\mathbf{R}) / k_{B} T}\left|\mu_{f i}(\mathbf{R})\right|^{2} \delta\left(\omega-\omega_{f i}(\mathbf{R})\right) d \mathbf{R}}{\int e^{-V_{i}(\mathbf{R}) / k_{B} T} d \mathbf{R}}$,

where $\omega_{f i}(\mathbf{R})=\left(V_{f}(\mathbf{R})-V_{i}(\mathbf{R})\right) / \hbar, \quad V_{i}(\mathbf{R})$ is the potentialenergy function in the ground electronic state whose averaged value $\frac{1}{2} E_{i \nu}$ appears in the quantum case as given in Eq. (1). ${ }^{10}$ For a comparison with this classical equation, the quantum mechanical one in Eq. (1) should have an extra factor of $\left(1-e^{-\hbar \omega / k_{B} T}\right)$ to include emission in the net absorption. ${ }^{16}$ In the classical limit, $\hbar \rightarrow 0$, the coefficient with this added factor reduces to that in Eq. $(5) .{ }^{17} V_{i}(\mathbf{R}), V_{f}(\mathbf{R})$ is dependent upon the electronic nature of the molecule alone and is unaffected by isotopic substitution. The same remark applies to $\sigma_{\text {total }}$ with the classical distribution. In the classical Franck-Condon approximation, since the momenta cancel in the expression for the absorption, the thermal distribution of the momenta at a given temperature does not affect the absorption.

\section{Three-isotope plot for high conversions}

When the photodissociation reaction proceeds to a high percent conversion to products, the result in Eq. (4a) can still be applied to obtain the relative amounts of different isotopomers remaining undissociated after time $t$. By treating photodissociation as a first-order rate process, the concentration $Q^{A}(t)$ of isotopomer $A$ remaining at time $t$ decays because of the photodissociation at $\omega$ as $Q^{A}(t)$ $=Q^{A}(0) \exp \left(-J^{A}(\omega) t\right)$. The relative amount of isotopomer $A^{\prime}$ remaining at time $t$ can be described by a function $\delta^{A^{\prime}, A}(\omega)$ defined by $1+\delta^{A^{\prime}, A}(\omega)=\left(\left(Q^{A^{\prime}}(t) / Q^{A^{\prime}}(0)\right) /\left(Q^{A}(t) / Q^{A}(0)\right)\right)$ $=\exp \left(-\left(J^{A^{\prime}}-J^{A}\right) t\right)$. Thereby, $\ln \left(1+\delta^{A^{\prime}, A}(\omega)\right)=-\left(J^{A^{\prime}}-J^{A}\right) t$. The relative amount of isotopomer $A^{\prime}$ surviving, considering the dissociation for the given range of frequencies $\omega$ of the light source, can be obtained by weighting the contribution from each $\omega$ by $I(\omega) \sigma^{(A)}(\omega)$,

$$
\begin{aligned}
\left\langle\ln \left(1+\delta^{A^{\prime}, A}\right)\right\rangle & =\frac{\int I(\omega) \sigma^{(A)}(\omega) \ln \left(1+\delta^{A^{\prime}, A}(\omega)\right) d \omega}{\int I(\omega) \sigma^{(A)}(\omega) d \omega} \\
& =-\frac{\int I(\omega) \sigma^{(A)}(\omega)\left(J^{A^{\prime}}-J^{A}\right) t d \omega}{\int I(\omega) \sigma^{(A)}(\omega) d \omega} .
\end{aligned}
$$

A photodissociation reaction with broadband absorption and high conversions thus yields a ratio of the relative amounts of isotopomers $A^{\prime}, A^{\prime \prime}$ remaining at time $t$ as

$$
\begin{aligned}
S & =\frac{\left\langle\ln \left(\left(Q^{A^{\prime \prime}}(t) / Q^{A^{\prime \prime}}(0)\right) /\left(Q^{A}(t) / Q^{A}(0)\right)\right)\right\rangle}{\left\langle\ln \left(\left(Q^{A^{\prime}}(t) / Q^{A^{\prime}}(0)\right) /\left(Q^{A}(t) / Q^{A}(0)\right)\right)\right\rangle} \\
& =\frac{\left\langle\ln \left(1+\delta^{A^{\prime \prime}, A}\right)\right\rangle}{\left\langle\ln \left(1+\delta^{A^{\prime}, A}\right)\right\rangle}=\frac{\int I(\omega) \sigma^{(A)}(\omega)\left(J^{A^{\prime \prime}}-J^{A}\right) t d \omega}{\int I(\omega) \sigma^{(A)}(\omega)\left(J^{A^{\prime}}-J^{A}\right) t d \omega} \\
& =\frac{\int I(\omega) \sigma^{(A)}(\omega) \epsilon^{\left(A^{\prime \prime}, A\right)} d \omega}{\int I(\omega) \sigma^{(A)}(\omega) \epsilon^{\left(A^{\prime}, A\right)} d \omega} .
\end{aligned}
$$

This $S$ is the slope of a plot of $\left\langle\ln \left(1+\delta^{A^{\prime \prime}, A}\right)\right\rangle$ vs $\langle\ln (1$ $\left.\left.+\delta^{A^{\prime}, A}\right)\right\rangle$, where the points are taken from different conversions (different reaction times). The value of $S$ from Eq. (7) is seen to be independent of $t$. Using Eq. (4b), $S=\beta$. That is, the slope of the plot based on different conversions of reactants is equal to the slope of the plot obtained using the values from different frequencies as the points on the plot.

\section{General comments}

A merit of three-isotope plots is that the slope obtained in the plot is dependent on the isotopic masses and the process that leads to the mass-dependent fractionation, without emphasizing many details of the process. For example, in the case of photodissociation the three-isotope plot only highlights the functional dependence of the absorption cross section $\sigma$ on the reciprocal masses $1 / m_{i}$. Even though the potential-energy and dipole moment surfaces are needed for the exact calculation of the absorption cross section, the three-isotope plot becomes independent of these quantities to a good approximation.

Sometimes, at negligible conversions, the rates of reaction of the three isotopomers are plotted as $\ln \left(\sigma^{A^{\prime \prime}} / \sigma^{A}\right)$ vs $\ln \left(\sigma^{A^{\prime}} / \sigma^{A}\right)$ for various frequencies. ${ }^{18}$ However, it should be noted that this logarithmic form obtained from different frequencies and used for small conversions is different from the logarithmic form obtained from different times and high conversions discussed in Sec. II C. We have given arguments [Eq. (7)] showing that for small conversions the slope $\alpha$ of the linear plot, rather than the corresponding log-log plot, is equal to the slope $S$ of the $\log -\log$ plot at high conversions. The equality is not based on perturbation theory.

The analysis thus far is applicable when the photodissociation process is spin allowed and the factors governing the rate of the photodissociation in different isotopomers are the mass-dependent frequencies and the normal coordinates. Photodissociation may also involve spin-forbidden processes, such as singlet-triplet transitions that become more allowed with a small probability due to, for example, a spinorbit coupling perturbation in the Hamiltonian or an electron-spin-nuclear-spin coupling (hyperfine coupling). The rates of such reactions for isotopes of odd atomic masses with a nonzero nuclear spin and even atomic masses with a zero nuclear spin are different. ${ }^{19,20}$ In such spin-forbidden photodissociation reactions, the isotopic fractionation de- 
pends on spin as well as mass. Thus, there can then be unusually high-isotopic fractionations. ${ }^{19,20}$ Also, in a threeisotope plot a slope of opposite sign compared to that for the regular mass-dependent processes also becomes possible. The result of the present work is not applicable to such spinforbidden cases. However, the fractionation of the two spinzero isotopomers can be calculated using the present method.

\section{E. Perturbation method applied to other processes}

The final result for photodissociation, Eq. (4a), is the same as that found for equilibrium chemical exchange reactions to first order. ${ }^{1,21}$ Bigeleisen and Mayer pointed out that in a chemical separation of isotopes, the fractionation is a quantum effect. ${ }^{1}$ The ratio of classical partition functions is unity, apart from any symmetry factors. Treating the rotational and translational partition functions classically and the vibrational partition function quantum mechanically, they obtained $^{22}$ a ratio of partition functions,

$$
\begin{aligned}
\frac{s}{s^{\prime}} f & =\prod_{i} \frac{u_{i}}{u_{i}^{\prime}} \frac{e^{-u_{i} / 2} /\left(1-e^{-u_{i}}\right)}{e^{-u_{i}^{\prime} / 2} /\left(1-e^{-u_{i}^{\prime}}\right)}=\frac{F\left(u_{i}\right)}{F\left(u_{i}^{\prime}\right)} \\
& \approx 1+\sum_{i} \frac{\Delta\left(u_{i}^{2}\right)}{24}
\end{aligned}
$$

where $s$ and $s^{\prime}$ are the symmetry numbers for the isotopomers, $F\left(u_{i}\right)=\Pi_{i} u_{i} e^{-u_{i} / 2} /\left(1-e^{-u_{i}}\right), u_{i}=\hbar \omega_{i} / k_{B} T$, where $\omega_{i}$ is the frequency of the $i$ th vibrational mode, and $u_{i}^{\prime}$ is defined similarly for the other isotopomer. The approximation in Eq. (8b) is valid for small $u_{i}$ or at high temperatures. Their analysis then uses the invariance of trace of the characteristic matrix to find the ratios of partition functions in terms of the isotopic masses. Alternatively, following exactly the method we have used in deriving Eq. (4a), we see from Eq. (8a) that the partition function depends upon the normal-mode frequencies $u_{i}$, and these $u_{i}$ are the eigenvalues of the characteristic matrix which depends on masses in the form $1 / m_{i}$, i.e., $u_{i}=u_{i}\left(1 / m_{1}, 1 / m_{2}, \ldots\right)$ and so $F\left(1 / m_{1}, 1 / m_{2}, \ldots, 1 / m_{i}, \ldots\right) / F\left(1 / m_{1}, 1 / m_{2}, \ldots, 1 / m_{i}^{\prime}, \ldots\right)$ $\approx 1+\left(\partial F / \partial\left(1 / m_{i}\right)\right) \Delta\left(1 / m_{i}\right)$. Thus we recover the $1 / m$ $-1 / m^{\prime}$ rule for the chemical equilibrium reaction fractionation. In deriving this result, we have avoided the hightemperature approximation, used in Bigeleisen and Mayer. So, to first order in $\Delta(1 / m)$, our result is independent of temperature.

When a different type of mass-dependent process is involved the functional dependence on $m$ can differ, as noted earlier in the introduction. For example, if the ratio of condensation rates of a molecule $A B$ onto a surface was proportional to $1 / \sqrt{m_{A B}}$, where $m_{A B}$ is the mass of the molecule, then the slope of a three-isotope fractionation plot would be $\left(1 / \sqrt{m_{A^{\prime} B}}-1 / \sqrt{m_{A B}}\right) /\left(1 / \sqrt{m_{A^{\prime \prime} B}}-1 / \sqrt{m_{A B}}\right)$ to first order in mass differences. This value differs somewhat from the value given in Eq. (4a), because of the difference in the nature of isotopic mass dependence of the rate process.

\section{NUMERICAL AND ANALYTICAL EXAMPLE: $\mathrm{N}_{\mathbf{2}} \mathrm{O}$ PHOTOLYSIS}

Nitrous oxide $\left(\mathrm{N}_{2} \mathrm{O}\right)$ is of considerable atmospheric interest since it is an efficient greenhouse gas, and we select it as an example. In the stratosphere, the photodissociation reaction $\mathrm{N}_{2} \mathrm{O}+\hbar \omega \rightarrow \mathrm{N}_{2}\left({ }^{1} \Sigma^{+}\right)+\mathrm{O}\left({ }^{1} D\right)$ is the most prominent mechanism for the removal of 446 (abbreviation for ${ }^{14} \mathrm{~N}^{14} \mathrm{~N}^{16} \mathrm{O}$ ) and its isotopomers 447, 448, 456, and $546 .{ }^{23}$ The principal range of absorption 175-215 nm corresponds to a singlet-singlet transition.

In Ref. 9 detailed computations for the wavelengthdependent absorption cross sections of the isotopomers and hence the fractionation factors were presented using the multidimensional reflection principle and the $a b$ initio potentialenergy surfaces and transition dipole moment function obtained from Ref. 24. To find $\left|\Psi_{\nu}(\mathbf{Q})\right|^{2} d \mathbf{Q}$ we first determined the normal-mode coordinates and frequencies with the procedure $^{15}$ summarized in Appendices B and C of Ref. 9, using the inertia matrix $G^{-1}$ and the force-constant matrix $F$ as defined in Ref. 15 and then finding the eigenvectors of the $G F$ matrix. The element $G_{\theta \theta}$ corresponding to the inertia for the bending vibration, not defined in Ref. 15, was obtained from Ref. 25. We expressed the harmonic-oscillator wave function in terms of the mass-dependent normal-mode coordinates $\mathbf{Q}$ and vibrational frequencies, and $\mathbf{Q}$ was then transformed to $\mathbf{R}$ with the appropriate transformation matrix that depends on isotopic substitution. ${ }^{9,15}$ The force constants obtained from Ref. 26 were used to form the force-constant matrix $F$. Using the results from those detailed calculations the fractionation factors $\epsilon^{447}$ and $\epsilon^{448}$ were obtained as functions of wavelength. In the wavelength range over which calculations were made, these fractionation factors were found to be approximately in the ranges $(-0.05,0.005)-$ $(-0.1,0.01)$, respectively, with the zero of fractionation near the absorption maximum, $185 \mathrm{~nm}$. In Ref. 9 calculations were also made for the other isotopomers of $\mathrm{N}_{2} \mathrm{O}$ : 456, 546, and 556 and comparison with the extensive experimental data on fractionation was given. Oddly, experimental data on the 447 spectral absorption seem to have been not published, even though 447 is commonly used in atmospheric analysis.

The emphasis of the current work is on the relation between $\epsilon^{447}(\omega)$ and $\epsilon^{448}(\omega)$ plotted using results for the different frequencies. A plot of $\epsilon^{448}$ vs $\epsilon^{447}$ is given in Fig. 1, each point obtained from Ref. 9 by evaluating both the fractionation factors 447 and 448 at the same wavelength, using the results from the different wavelengths. This linear plot gives a slope of approximately 0.532 with a zero intercept in the temperature range of 233-300 K. The slope for the fractionation by photolysis, is close to the perturbation slope $\left(1 / m_{O^{16}}-1 / m_{O^{17}}\right) /\left(1 / m_{O^{16}}-1 / m_{O^{18}}\right)=0.529$ for the massdependent fractionation, obtained in the present perturbation analysis. They are not precisely the same, since one is 0.532 and the other is 0.529 , because the terms of higher order in $\Delta(1 / m)$ that are present in the computational result are absent in the perturbation treatment.

Using the computational results, we found that the slope of the linear plot $\epsilon^{448}(\omega)$ vs $\epsilon^{447}(\omega)$ was 0.5325 at $233 \mathrm{~K}$ and 0.5338 at $297 \mathrm{~K}$, while for $\ln \left(\sigma^{448}(\omega) / \sigma^{446}(\omega)\right)$ vs 


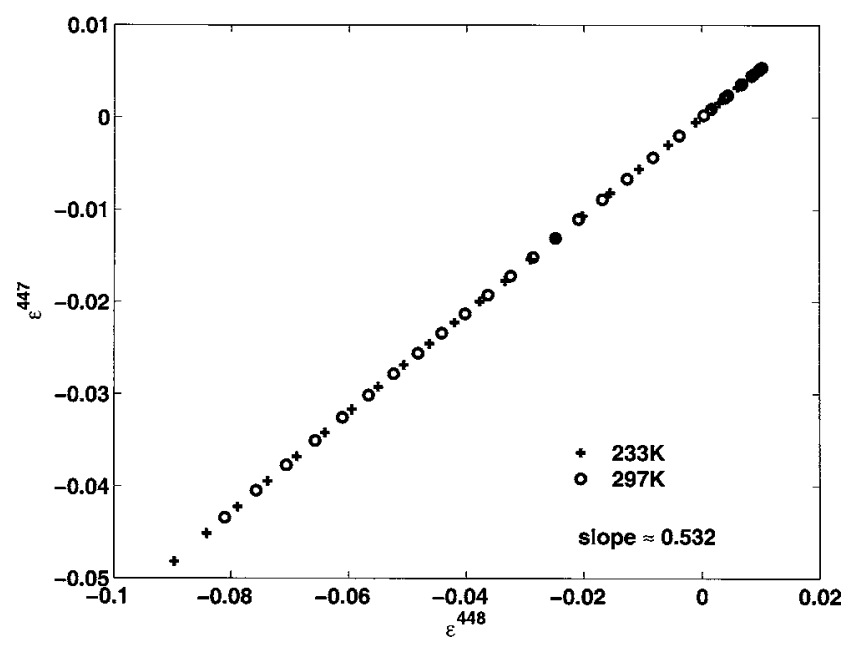

FIG. 1. Three-isotope plot of oxygen isotopes of $\mathrm{N}_{2} \mathrm{O}$.

$\ln \left(\sigma^{447}(\omega) / \sigma^{446}(\omega)\right)$ plot the slopes were 0.5248 and 0.5250 , respectively, so indicating that both plots are relatively unaffected by temperature, although the log-log plot is slightly less affected. The present results for $233-300 \mathrm{~K}$ show that the slope is nearly the same over this temperature range. At even lower temperatures the contribution to absorption comes significantly from the ground vibrational state of the molecule alone and so the coefficients of the powers of $\Delta(1 / m)$ will depend weakly on temperature. So we expect that the slopes do not change much at lower temperatures, even though one is far from the high-temperature regime.

Because of the close comparison between massdependent values obtained using computed fractionation factors and the theoretical analysis with no computation, the importance of the perturbation theoretical result for the $\mathrm{N}_{2} \mathrm{O}$ test case becomes apparent. The small variation of the absorption cross section with the mass, as indicated by the fractionation factors less than 0.1 , justifies the use of perturbation expansion in $\sigma$ to the first order.

From the data on $\mathrm{N}_{2} \mathrm{O}$ fractionation in unspecified processes different from photodissociation, a slope of 0.515 was obtained $^{27}$ for a three-isotope plot of $\epsilon^{448}$ vs $\epsilon^{447}$. In their later experiments, Cliff et al. ${ }^{28}$ observed an excess of $\mathrm{O}^{17}$ when the samples from lower stratosphere were analyzed using this slope of 0.515. This observation called for an accurate estimate of the three-isotope plot slope obtained by photolysis. An assumed slope of 0.515 for photolysis required additional $\mathrm{N}_{2} \mathrm{O}$ sources/sinks to explain the observation. Kaiser et al. ${ }^{18}$ used a slope of about 0.520 obtained from the broadband photolysis experiments by Rockmann et $a .^{29}$ The measured value of 447 fractionation factor is not published in Ref. 29, and slopes between 0.50 and 0.53 are possible with the published data on $448 .^{30}$ More refined experiments $^{31}$ do not give the measured value of 447 fractionation factor nor the slope of the three-isotope plot. Other theoretical estimates for this slope 0.525 and 0.517 from Refs. 32 and 33 using detailed quantum-mechanical wave packet propagation on the potential-energy surface also could not help resolve the debate about the interpretation of the $\mathrm{O}^{17}$ excess in atmospheric samples. ${ }^{28}$ The slope 0.532 we obtained in a linear plot of fractionation factors from the detailed computation, and supported by the almost similar perturbation-theory result, should, we believe, be used as the mass-dependent slope standard for the $\mathrm{N}_{2} \mathrm{O}$ photodissociation. We also believe that this slope of 0.532 should be included in a chemical transport model similar to that of McLinden et ll $^{34}$ to see what fraction of the current discrepancy in the atmospheric measurements of Ref. 28 can be accounted for solely by photodissociation. A decision could then be made whether a search for additional sources/sinks of $\mathrm{N}_{2} \mathrm{O}$ is needed.

\section{CONCLUSIONS}

Using a perturbation theoretical analysis an expression is obtained for the slope of three-isotope plots in photolytic fractionation. The result obtained here is expected to be a good approximation to analyze the mass-dependent effects in the photolytic process when the fractionation factors are small, justifying a first-order perturbation in the absorption cross section. For $\mathrm{N}_{2} \mathrm{O}$, the result obtained by the present analysis is a close match with that of detailed computational calculations ${ }^{9}$ of photodissociation fractionation. The comparison of the linear and log-log plots is discussed in the context of small and large conversions. This perturbation method given here for photodissociation reactions can be extended to some other kinetic processes once the functional form in which the masses appear in the equation for fractionation is known.

\section{ACKNOWLEDGMENT}

It is a pleasure to acknowledge the support of this research by the National Science Foundation.

${ }^{1}$ J. Bigeleisen and M. G. Mayer, J. Chem. Phys. 15, 261 (1947).

${ }^{2}$ H. C. Urey, J. Chem. Soc. 1947, 562.

${ }^{3}$ Y. Matsuhisa, J. R. Goldsmith, and R. N. Clayton, Geochim. Cosmochim. Acta 42, 173 (1978).

${ }^{4}$ R. N. Clayton, L. Grossman, and T. K. Mayeda, Science 182, 485 (1973).

${ }^{5}$ M. H. Thiemens and J. E. Heidenreich, Science 219, 1073 (1983).

${ }^{6}$ C. A. M. Brenninkmeijer, C. Janssen, J. Kaiser, T. Rockmann, T. S. Rhee, and S. S. Assonov, Chem. Rev. (Washington, D.C.) 103, 5125 (2003), and references cited therein.

${ }^{7}$ R. E. Weston, Chem. Rev. (Washington, D.C.) 99, 2115 (1999), and references cited therein.

${ }^{8}$ E. D. Young, A. Galy, and H. Nagahara, Geochim. Cosmochim. Acta 66, 1095 (2002)

${ }^{9}$ M. K. Prakash, J. D. Weibel, and R. A. Marcus, J. Geophys. Res., [Atmos.] (to be published).

${ }^{10}$ S. Y. Lee, R. C. Brown, and E. J. Heller, J. Phys. Chem. 87, 2045 (1983).

${ }^{11}$ R. Schinke, Photodissociation Dynamics (Cambridge University Press, New York, 1993).

${ }^{12} \mathrm{~A}$ comparison of the absorption cross sections associated with direct, nearly direct, and indirect photodissociation is given in Fig. 1.6 of Ref. 11.

${ }^{13}$ E. J. Heller, J. Chem. Phys. 68, 2066 (1978).

${ }^{14}$ Y. L. Yung and C. E. Miller, Science 278, 1778 (1997).

${ }^{15}$ S. Califano, Vibrational States (Wiley, New York, 1976).

${ }^{16}$ D. A. McQuarrie, Statistical Mechanics (University Science, Sausalito, CA, 2000).

${ }^{17}$ P. H. Berens and K. R. Wilson, J. Chem. Phys. 74, 4872 (1981).

${ }^{18}$ J. Kaiser, T. Rockmann, and C. A. M. Brenninkmeijer, J. Geophys. Res. 109, D03305 (2004). 
${ }^{19}$ E. M. Galimov, Geokhimiya 1979, 274 [Geochem. Internatl. 1979, 155].

${ }^{20}$ S. E. Bhattacharya, J. Savarino, and M. H. Thiemens, Geophys. Res. Lett. 27, 1459 (2000).

${ }^{21}$ J. Bigeleisen, J. Chem. Phys. 23, 2264 (1955).

${ }^{22}$ The partition function given in Eqs. (8a) and (8b) is from Eqs. (10) and (11b) in Ref. 1.

${ }^{23}$ K. Minschwaner, R. J. Salawitch, and M. B. McElroy, J. Geophys. Res. 98, 10543 (1993).

${ }^{24}$ M. N. Daud, G. G. Balint-Kurti, and A. Brown, J. Chem. Phys. 122 054305 (2005). Potential-energy and dipole moment surfaces are available at ftp://ftp.aip.org/epaps/journ_chem_phys/E-JCPSA6-122-303502/

${ }^{25}$ S. M. Ferigle and A. G. Meister, J. Chem. Phys. 19, 982 (1951).

${ }^{26}$ A. G. Csaszar, J. Phys. Chem. 98, 8823 (1994).

${ }^{27}$ S. S. Cliff and M. H. Thiemens, Science 278, 1774 (1997).

${ }^{28}$ S. S. Cliff, C. A. M. Brenninkmeijer, and M. H. Thiemens, J. Geophys. Res. 104, 16171 (1999).
${ }^{29}$ T. Rockmann, J. Kaiser, C. A. M. Brenninkmeijer, J. N. Crowley, R. Borchers, W. A. Brand, and P. J. Crutzen, J. Geophys. Res., [Atmos.] 106, 10403 (2001)

${ }^{30}$ The measured data on 447 were not published in Ref. 29 and 448 fractionation factors relative to 446 were $34.2 \pm 0.8$. If the 0.520 was calculated using the mean value 34.2 per mil given for 448 in Ref. 29, then with the error bar of \pm 0.8 per mil, the slope can have any value between 0.50 and 0.53

${ }^{31}$ J. Kaiser, Ph.D. thesis, Johannes Gutenberg University, Mainz, Germany, 2002.

${ }^{32}$ M. S. Johnson, G. D. Billing, A. Gruodis, and M. H. M. Janssen, J. Phys. Chem. A 105, 8672 (2001)

${ }^{33}$ S. Nanbu and M. S. Johnson, J. Phys. Chem. A 108, 8905 (2004).

${ }^{34}$ C. A. McLinden, M. J. Prather, and M. S. Johnson, J. Geophys. Res., [Atmos.] 108, 4233 (2003). 